TRANSACTIONS OF THE

AMERICAN MATHEMATICAL SOCIETY

Volume 363, Number 3, March 2011, Pages 1183-1195

S 0002-9947(2010)04869-X

Article electronically published on October 21, 2010

\title{
NONSYMMETRIC CONICAL UPPER DENSITY AND $k$-POROSITY
}

\author{
ANTTI KÄENMÄKI AND VILLE SUOMALA
}

\begin{abstract}
We study how the Hausdorff measure is distributed in nonsymmetric narrow cones in $\mathbb{R}^{n}$. As an application, we find an upper bound close to $n-k$ for the Hausdorff dimension of sets with large $k$-porosity. With $k$-porous sets we mean sets which have holes in $k$ different directions on every small scale.
\end{abstract}

\section{INTRODUCTION}

It is a well-known fact that for a set $A \subset \mathbb{R}^{n}$ with finite $s$-dimensional Hausdorff measure, $\mathcal{H}^{s}(A)<\infty$, we have

$$
1 \leq \limsup _{r \downarrow 0} \frac{\mathcal{H}^{s}(A \cap B(x, r))}{r^{s}} \leq 2^{s}
$$

for $\mathcal{H}^{s}$-almost every $x \in A$. For a proof, see, for example, [12, Theorem 6.2(1)]. This is analogous to the classical Lebesgue Density Theorem. Using this fact, we know roughly how much of $A$ there is in small balls. Mattila [11 studied how $A$ is distributed in such balls. He was able to estimate how much of $A$ there is near $(n-m)$-planes. More precisely, assuming $0 \leq m<s \leq n$ and denoting

$$
\begin{aligned}
X(x, V, \alpha) & =\left\{y \in \mathbb{R}^{n}: \operatorname{dist}(y-x, V)<\alpha|y-x|\right\}, \\
X(x, r, V, \alpha) & =X(x, V, \alpha) \cap B(x, r),
\end{aligned}
$$

as $x \in \mathbb{R}^{n}, V \in G(n, m), r>0$, and $0<\alpha \leq 1$, he proved that there exists a constant $c=c(n, m, s, \alpha)>0$ such that

$$
\limsup _{r \downarrow 0} \inf _{V \in G(n, n-m)} \frac{\mathcal{H}^{s}(A \cap X(x, r, V, \alpha))}{r^{s}} \geq c
$$

for $\mathcal{H}^{s}$-almost every $x \in A$ whenever $A \subset \mathbb{R}^{n}$ is such that $\mathcal{H}^{s}(A)<\infty$. Here $G(n, m)$ denotes the collection of all $m$-dimensional linear subspaces of $\mathbb{R}^{n}$; see [12, $\S 3.9]$. Actually (1.2) is just a special case of Mattila's result, as his theorem can be applied also for more general cones; see [11, Theorem 3.3].

Received by the editors May 1, 2004 and, in revised form, July 4, 2008.

2000 Mathematics Subject Classification. Primary 28A75; Secondary 28A78, 28A80.

Key words and phrases. Conical density, porosity, Hausdorff dimension.

The first author acknowledges the support of the Academy of Finland (project \#114821).

(C)2010 American Mathematical Society Reverts to public domain 28 years from publication 
In Theorem 2.5 we show that if $A$ is as above, then it cannot be concentrated in very small regions, not even inside the cones $X(x, r, V, \alpha)$. More precisely, denoting

$$
\begin{aligned}
H(x, \theta) & =\left\{y \in \mathbb{R}^{n}:(y-x) \cdot \theta>0\right\}, \\
H(x, \theta, \eta) & =\left\{y \in \mathbb{R}^{n}:(y-x) \cdot \theta>\eta|y-x|\right\},
\end{aligned}
$$

for $x \in \mathbb{R}^{n}, \theta \in S^{n-1}$, and $0<\eta \leq 1$, we prove under the same assumptions as in (1.2) that there exists a constant $c=c(n, m, s, \alpha, \eta)>0$ such that

$$
\limsup \inf _{r \downarrow 0} \frac{\mathcal{H}^{s}(A \cap X(x, r, V, \alpha) \backslash H(x, \theta, \eta))}{r^{s}} \geq c
$$

for $\mathcal{H}^{s}$-almost every $x \in A$. Here $S^{n-1}$ denotes the unit sphere of $\mathbb{R}^{n}$. To help the geometric visualization, it might be helpful to take $\alpha$ and $\eta$ close to 0 and $\theta \in V \cap S^{n-1}$. Our method also gives a more elementary proof for (1.2) and it can also be used to obtain similar results for more general measures; see Theorem 2.7 .

The nonsymmetric conical upper density theorem is essential in our application to $k$-porous sets, that is, the sets with por $_{k}>0$; see (1.5). The notation of porosity, or 1-porosity using our terminology, has arisen from the study of dimensional estimates related, for example, to the boundary behavior of quasiconformal mappings. See Koskela and Rohde [9, Martio and Vuorinen [10], Sarvas [15], Trocenko [17], and Väisälä [18. The dimensional properties of 1-porous sets are well known. Using a version of (1.2), Mattila showed that if porosity is close to its maximum value $\frac{1}{2}$, then the dimension cannot be much larger than $n-1$. More precisely,

$$
\sup \left\{s>0: \operatorname{por}_{1}(A)>\varrho \text { and } \operatorname{dim}_{\mathrm{H}}(A)>s \text { for some } A \subset \mathbb{R}^{n}\right\} \longrightarrow n-1
$$

as $\varrho \rightarrow \frac{1}{2}$. Here $\operatorname{dim}_{\mathrm{H}}$ refers to the Hausdorff dimension. Later Salli [14] generalized this result for the Minkowski dimension, and found the correct asymptotics. The concept of 1-porosity has also been generalized for measures, and it leads to a similar kind of dimension bounds. See Järvenpää and Järvenpää [4] and the references therein.

Motivated by the fact that each $V \in G(n, n-1)$ has maximal 1-porosity, we introduce a porosity condition which also describes sets whose dimension is smaller than $n-1$. For any integer $0<k \leq n, x \in \mathbb{R}^{n}, A \subset \mathbb{R}^{n}$, and $r>0$ we set

$$
\begin{array}{r}
\operatorname{por}_{k}(A, x, r)=\sup \left\{\varrho: \text { there are } z_{1}, \ldots, z_{k} \in \mathbb{R}^{n}\right. \text { such that } \\
\qquad\left(z_{i}, \varrho r\right) \subset B(x, r) \backslash A \text { for every } i, \\
\text { and } \left.\left(z_{i}-x\right) \cdot\left(z_{j}-x\right)=0 \text { for } i \neq j\right\} .
\end{array}
$$

Here $\cdot$ is the inner product. The $k$-porosity of $A$ at a point $x$ is defined to be

$$
\operatorname{por}_{k}(A, x)=\underset{r \downarrow 0}{\liminf } \operatorname{por}_{k}(A, x, r),
$$

and the $k$-porosity of $A$ is given by

$$
\operatorname{por}_{k}(A)=\inf _{x \in A} \operatorname{por}_{k}(A, x) .
$$

This means that $k$-porous sets have holes in $k$ orthogonal directions near each of its points in every small scale. We shall now give a concrete example where $k$-porosity occurs naturally. Suppose $0<\lambda<\frac{1}{2}$ and let $C_{\lambda} \subset \mathbb{R}$ be the usual $\lambda$-Cantor set; see [12, §4.10]. It is clearly a 1 -porous set with $\operatorname{por}_{1}\left(C_{\lambda}\right) \approx \frac{1}{2}-\lambda$. Mattila's result (1.3) implies that $\operatorname{dim}_{\mathrm{H}}\left(C_{\lambda}\right) \rightarrow 0$ as $\operatorname{por}_{1}\left(C_{\lambda}\right) \rightarrow \frac{1}{2}$. Of course, we could obtain the same information just by calculating the Hausdorff dimension of 
the self-similar set $C_{\lambda}$ and letting $\lambda \rightarrow 0$, but our aim was to provide the reader with an illustrative example. The sets $C_{\lambda} \times C_{\lambda} \subset \mathbb{R}^{2}$ and $C_{\lambda} \times C_{\lambda} \times[0,1] \subset \mathbb{R}^{3}$ are clearly 2 -porous with por $_{2} \approx \frac{1}{2}-\lambda$. For these sets (1.3) does not give any reasonable dimension bound. However, it would be desirable to see, also in terms of porosity, that $\operatorname{dim}_{\mathrm{H}}\left(C_{\lambda} \times C_{\lambda}\right) \rightarrow 0$ and $\operatorname{dim}_{\mathrm{H}}\left(C_{\lambda} \times C_{\lambda} \times[0,1]\right) \rightarrow 1$ as $\lambda \rightarrow 0$. This follows as an immediate application of Theorem 3.2 Using our nonsymmetric conical upper density theorem, we show that

$$
\sup \left\{s>0: \operatorname{por}_{k}(A)>\varrho \text { and } \operatorname{dim}_{\mathrm{H}}(A)>s \text { for some } A \subset \mathbb{R}^{n}\right\} \longrightarrow n-k
$$

as $\varrho \rightarrow \frac{1}{2}$. Observe also that in the proof of Theorem 3.2 the orthogonality in (1.4) plays no role and we may replace it by an assumption of a uniform lower bound for the angles between $z_{i}-x$ and the $(k-1)$-plane spanned by vectors $z_{j}-x, i \neq j$.

Let us now discuss the situation when porosity is small. It is well known (for example, see [10]) that if $A \subset \mathbb{R}^{n}$ with $\operatorname{por}_{1}(A, x, r) \geq \varrho>0$ for all $x \in A$ and $0<r<r_{0}$, then

$$
\operatorname{dim}_{\mathrm{M}}(A)<n-c \varrho^{n},
$$

where $c>0$ depends only on $n$, and $\operatorname{dim}_{M}$ refers to the Minkowski dimension; see [12, §5.3]. It might be possible to get a better estimate if por $_{1}$ is replaced by por $_{k}$ for some $k>1$, but this condition does not feel very natural if the size of the holes is small. However, if $V \in G(n, m)$ is fixed and the condition $\operatorname{por}_{1}(A, x, r) \geq \varrho$ is replaced by

$$
\sup \left\{\varrho^{\prime}: B\left(z, \varrho^{\prime} r\right) \subset B(x, r) \backslash A \text { for some } z \in V+\{x\}\right\} \geq \varrho,
$$

then $n$ in (1.6) can be replaced by $m$; see Theorem 4.3. This is a rather immediate consequence of (1.6), but our main point is to give a simple proof for (1.6) using iterated function systems.

\section{NonsymmetriC CONICAL UPPER DENSITY}

We shall first prove a density theorem for nonsymmetric regions and then prove our main theorem by using a similar argument on $(n-m)$-planes. The proofs rely on the following geometric fact.

Lemma 2.1. For given $0<\beta<\pi$, there is $q=q(n, \beta) \in \mathbb{N}$ such that in any set of $q$ points in $\mathbb{R}^{n}$, there are always three points which determine an angle between $\beta$ and $\pi$.

Remark 2.2. Erdös and Füredi [1] have shown that for the smallest possible choice of $q$ it follows that

$$
2^{(\pi /(\pi-\beta))^{n-1}} \leq q(n, \beta) \leq 2^{(4 \pi /(\pi-\beta))^{n-1}}+1
$$

For the convenience of the reader we shall give below a different proof which establishes the existence of some such $q$. The estimate that we get here for $q$ is, however, quite bad compared to the best possible one.

Proof. Let $A$ be a set of points in $\mathbb{R}^{n}$ so that all angles formed by its points are less than $\beta$. Let us fix $0<\eta<1$ and cover $\mathbb{R}^{n} \backslash\{0\}$ by cones $C_{i}=H\left(0, \theta_{i}, \eta\right)$, $i \in\{1,2, \ldots, k\}$, where the constant $k=k(n, \eta) \in \mathbb{N}$ depends only on $n$ and $\eta$. To visualize the situation, note that if $\beta$ is close to $\pi$, then $\eta$ is close to 1 and cones $C_{i}$ are very narrow. To simplify the notation, we denote $C_{i, y}=C_{i}+\{y\}$ for $y \in \mathbb{R}^{n}$. 


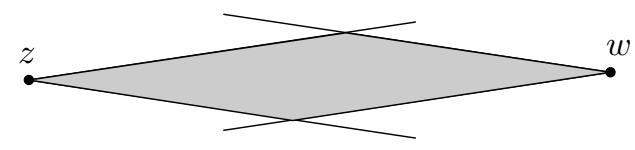

Figure A. All points lying on the gray region form a large angle with points $z$ and $w$.

For any index $i_{1} i_{2} \cdots i_{j}$, where $j \in \mathbb{N}$ and $i_{m} \in\{1,2, \ldots, k\}$ for $1 \leq m \leq j$, we define sets $A_{i_{1} i_{2} \cdots i_{j}}$ in the following way: We begin by fixing $x \in A$ and setting $A_{i}=A \cap C_{i, x}$ for $1 \leq i \leq k$. If $A_{i_{1} i_{2} \cdots i_{j}}$ has been defined, we choose $y \in A_{i_{1} i_{2} \cdots i_{j}}$ and define $A_{i_{1} i_{2} \cdots i_{j} l}=A_{i_{1} i_{2} \cdots i_{j}} \cap C_{l, y}$ for $1 \leq l \leq k$ (if $A_{i_{1} i_{2} \cdots i_{j}}$ is empty, then $A_{i_{1} i_{2} \cdots i_{j} l}$ is as well). We refer to $y$ as the corner of $A_{i_{1} i_{2} \cdots i_{j} l}$. It follows directly from the definition of the sets $A_{i_{1} i_{2} \cdots i_{j}}$ that

$$
\operatorname{card} A_{i_{1} i_{2} \cdots i_{j}} \leq 1+\sum_{l=1}^{k} \operatorname{card} A_{i_{1} i_{2} \cdots i_{j} l} .
$$

Iterating this, we get

$$
\operatorname{card} A \leq \sum_{j=0}^{k} k^{j}+\sum_{i_{1} i_{2} \cdots i_{k}} \sum_{l=1}^{k} \operatorname{card} A_{i_{1} i_{2} \cdots i_{k} l} .
$$

The main point of the proof is the observation that if $\eta=\eta(\beta)$ is chosen to be close enough to 1 in the beginning, then the following is true: If $z$ and $w$ are the corners of $A_{i_{1} i_{2} \cdots i_{j}}$ and $A_{i_{1} i_{2} \cdots i_{j} i_{j+1} \cdots i_{m}}$, respectively, and if $z \in C_{i_{m}, w}$, then $A \cap C_{i_{j}, z} \cap C_{i_{m}, w}=\emptyset$; see Figure $\mathrm{A}$. It follows by induction from the above fact that for given $A_{i_{1} i_{2} \cdots i_{j}}$ we have

$$
\operatorname{card}\left\{l: A_{i_{1} i_{2} \cdots i_{j} l} \neq \emptyset\right\} \leq k-j .
$$

In particular, $A_{i_{1} i_{2} \cdots i_{k+1}}=\emptyset$ for any choice of $i_{1} i_{2} \ldots i_{k+1}$. Combined with (2.1), this gives card $A \leq \sum_{j=0}^{k} k^{j}$. This number depends only on $k=k(n, \beta)$ and the claim follows.

For $0<\eta \leq 1$ we define

$$
\begin{aligned}
& t(\eta)=\sqrt{\frac{\eta^{2}+4}{\eta^{2}}}, \\
& \gamma(\eta)=\frac{1}{t(\eta)} .
\end{aligned}
$$

Notice that $t(\eta) \geq 2$ and $\eta / \sqrt{5} \leq \gamma(\eta) \leq \eta / 2$.

Lemma 2.3. Suppose that $y \in \mathbb{R}^{n}, \theta \in S^{n-1}, 0<\eta \leq 1, t=t(\eta)$, and $\gamma=\gamma(\eta)$. If $z \in \mathbb{R}^{n} \backslash(B(y, t r) \cup H(y, \theta, \gamma))$, then

$$
B(z, r) \cap H(y, \theta, \eta)=\emptyset .
$$

Proof. Take $w \in \mathbb{R}^{n}$ such that it maximizes $(w-y) \cdot \theta /|w-y|$ in the closure of $B(z, r)$. It suffices to prove that $(w-y) \cdot \theta /|w-y|<\eta$; see Figure B, It is straightforward 


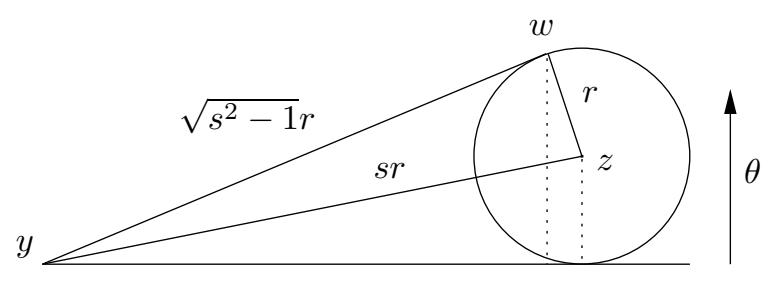

Figure B. Illustration for the proof of Lemma 2.3 ,

to check that $\eta \sqrt{s^{2}-1} \geq 1+\gamma s$ when $s \geq t$. Now denoting $s=|y-z| / r$, we have $s \geq t>1$ and thus

$$
\begin{aligned}
&(w-y) \cdot \theta<r+\gamma|y-z|=(1+\gamma s) r \\
& \leq \eta \sqrt{s^{2}-1} r=\eta|w-y|,
\end{aligned}
$$

which finishes the proof.

Theorem 2.4. Suppose $0<\eta \leq 1$ and $0<s \leq n$. Then there is a constant $c=c(n, s, \eta)>0$ such that

$$
\limsup _{r \downarrow 0} \inf _{\theta \in S^{n-1}} \frac{\mathcal{H}^{s}(A \cap B(x, r) \backslash H(x, \theta, \eta))}{r^{s}} \geq c
$$

for $\mathcal{H}^{s}$-almost every $x \in A$ whenever $A \subset \mathbb{R}^{n}$ with $\mathcal{H}^{s}(A)<\infty$.

Proof. Take $c>0$ and assume that there exists a Borel set $B \subset \mathbb{R}^{n}$ with $\mathcal{H}^{s}(B)>0$ such that for each $x \in B$ and $0<r<r_{0}$ there is $\theta \in S^{n-1}$ for which

$$
\mathcal{H}^{s}(B \cap B(x, r) \backslash H(x, \theta, \eta))<c r^{s} .
$$

It suffices to find a positive lower bound for $c$ in terms of $n, s$, and $\eta$.

Using (1.1), and replacing $B$ by a suitable subset if necessary, we may assume that

$$
\mathcal{H}^{s}(B \cap B(x, r))<2^{s+1} r^{s}
$$

for all $0<r<r_{0}$ and $x \in B$. Moreover, using the lower estimate of (1.1), we find that $0<r<r_{0} / 3$ and $x \in B$ such that

$$
\mathcal{H}^{s}(B \cap B(x, r))>\frac{1}{2} r^{s} .
$$

Set $t=t(\eta), \gamma=\gamma(\eta)$, and take $0<\delta<1$. Let us fix $\beta<\pi$ such that the opening angle of $H(x, \theta, \gamma)$ is smaller than $\beta$, and let $q=q(n, \beta)$ be as in Lemma 2.1. We may cover the set $B \cap B(x, r)$ by $4^{n} \delta^{-n}$ balls of radius $\delta r$ with centers in $B$. Using (2.4), we notice that there exists $x_{1} \in B \cap B(x, r)$ such that

$$
\mathcal{H}^{s}\left(B \cap B\left(x_{1}, \delta r\right)\right)>4^{-n} \delta^{n} 2^{-1} r^{s} .
$$

The set $B \cap B(x, r) \backslash B\left(x_{1}, t \delta r\right)$ can also be covered by $4^{n} \delta^{-n}$ balls of radius $\delta r$ with centers in $B$. Whence, using (2.3) and (2.4),

$$
\mathcal{H}^{s}\left(B \cap B(x, r) \backslash B\left(x_{1}, t \delta r\right)\right)>\left(\frac{1}{2}-2^{s+1} t^{s} \delta^{s}\right) r^{s} .
$$

If $\frac{1}{2}-2^{s+1} t^{s} \delta^{s}>0$, we find $x_{2} \in B \cap B(x, r) \backslash B\left(x_{1}, t \delta r\right)$ for which

$$
\mathcal{H}^{s}\left(B \cap B\left(x_{2}, \delta r\right)\right)>4^{-n} \delta^{n}\left(\frac{1}{2}-2^{s+1} t^{s} \delta^{s}\right) r^{s} .
$$


Choosing $\delta=\delta(n, s, \eta)>0$ small enough and continuing in this manner, we find $q$ points $x_{1}, \ldots, x_{q} \in B \cap B(x, r)$ with $\left|x_{i}-x_{j}\right| \geq t \delta r$ for $i \neq j$, such that for each $i \in\{1, \ldots, q\}$ we have

$$
\begin{aligned}
\mathcal{H}^{s}\left(B \cap B\left(x_{i}, \delta r\right)\right) & >4^{-n} \delta^{n}\left(\frac{1}{2}-(q-1) 2^{s+1} t^{s} \delta^{s}\right) r^{s} \\
& =: c(n, s, \eta)(3 r)^{s},
\end{aligned}
$$

where $c(n, s, \eta)>0$.

According to Lemma 2.1, we may choose three points $y, y_{1}, y_{2}$ from the set $\left\{x_{1}, \ldots, x_{q}\right\}$ such that for each $\theta \in S^{n-1}$ there is $i \in\{1,2\}$ for which $y_{i} \in$ $\mathbb{R}^{n} \backslash(B(y, t \delta r) \cup H(y, \theta, \gamma))$. We obtain, using Lemma 2.3, that for each $\theta \in S^{n-1}$ there is $i \in\{1,2\}$ such that

$$
B\left(y_{i}, \delta r\right) \subset B(y, 2(1+\delta) r) \backslash H(y, \theta, \eta) .
$$

Thus, applying (2.5), we have

$$
\mathcal{H}^{s}(B \cap B(y, 3 r) \backslash H(y, \theta, \eta))>c(n, s, \eta)(3 r)^{s}
$$

for all $\theta \in S^{n-1}$. Recalling (2.2), we conclude that $c \geq c(n, s, \eta)$. The proof is finished.

Theorem 2.5. Suppose $0<\alpha, \eta \leq 1$ and $0 \leq m<s \leq n$. Then there is a constant $c=c(n, m, s, \alpha, \eta)>0$ such that

$$
\limsup _{r \downarrow 0} \inf _{\substack{\theta \in S^{n-1} \\ V \in G(n, n-m)}} \frac{\mathcal{H}^{s}(A \cap X(x, r, V, \alpha) \backslash H(x, \theta, \eta))}{r^{s}} \geq c
$$

for $\mathcal{H}^{s}$-almost every $x \in A$ whenever $A \subset \mathbb{R}^{n}$ with $\mathcal{H}^{s}(A)<\infty$.

Proof. For any $V, W \in G(n, n-m)$, we set $d(V, W)=\sup _{x \in V \cap S^{n-1}} \operatorname{dist}(x, W)$. With this metric $G(n, n-m)$ is a compact metric space; see Salli [13. Defining for each $V \in G(n, n-m)$ a set $\{W: d(V, W)<\alpha / 2\}$ we notice that a finite number of these sets is still a cover. We assume that the sets assigned to the planes $V_{1}, \ldots, V_{l}$, where $l=l(n, m, \alpha)$, cover $G(n, n-m)$. For any $W$, it holds that $d\left(V_{i}, W\right)<\alpha / 2$ with some $i \in\{1, \ldots, l\}$. This implies $X\left(0, V_{i}, \alpha / 2\right) \subset X(0, W, \alpha)$. Thus, for each $W \in G(n, n-m)$, there is $i$ such that

$$
X(x, r, W, \alpha) \supset X\left(x, r, V_{i}, \alpha / 2\right)
$$

for all $r>0$ and $x \in \mathbb{R}^{n}$. We shall prove that if $A \subset \mathbb{R}^{n}$ with $\mathcal{H}^{s}(A)<\infty$, then

$$
\limsup _{r \downarrow 0} \inf _{\substack{\theta \in S^{n-1} \\ i \in\{1, \ldots, l\}}} \frac{\mathcal{H}^{s}\left(A \cap X\left(x, r, V_{i}, \alpha / 2\right) \backslash H(x, \theta, \eta)\right)}{r^{s}} \geq c(n, m, s, \alpha, \eta)
$$

for $\mathcal{H}^{s}$-almost every $x \in A$ from which the claim follows easily by using (2.6).

Take $c>0$ and assume that there is a Borel set $B \subset \mathbb{R}^{n}$ with $\mathcal{H}^{s}(B)>0$ such that for each $x \in B$ and $0<r<r_{0}$ there are $i$ and $\theta \in S^{n-1}$ for which

$$
\mathcal{H}^{s}\left(B \cap X\left(x, r, V_{i}, \alpha / 2\right) \backslash H(x, \theta, \eta)\right)<c r^{s} .
$$

According to (1.1) we may assume that

$$
\mathcal{H}^{s}(B \cap B(x, r))<2^{s+1} r^{s}
$$

for all $0<r<r_{0}$ and $x \in B$. Using the lower estimate of (1.1), we find that $0<r<r_{0} / 3$ and $x \in B$ such that

$$
\mathcal{H}^{s}(B \cap B(x, r))>\frac{1}{2} r^{s} .
$$




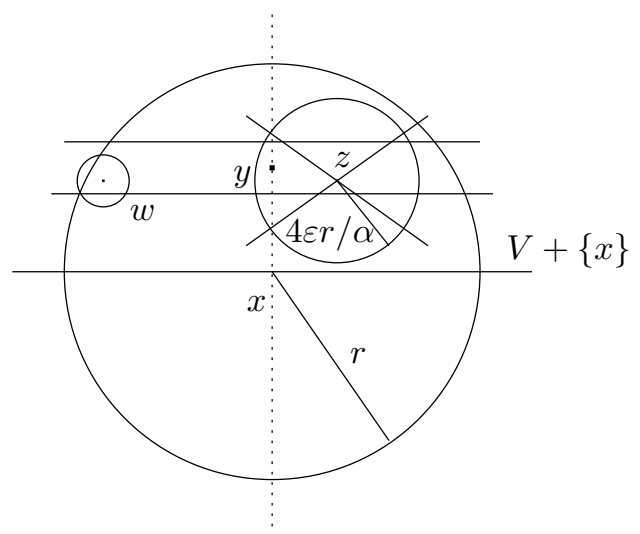

Figure C. Illustration for the proof of Theorem 2.5.

Next we define

$$
\begin{array}{r}
B_{i}=\left\{z \in B: \mathcal{H}^{s}\left(B \cap X\left(z, 3 r, V_{i}, \alpha / 2\right) \backslash H(z, \theta, \eta)\right)<c(3 r)^{s}\right. \\
\text { for some } \left.\theta \in S^{n-1}\right\} .
\end{array}
$$

Since $\bigcup_{i=1}^{l} B_{i}=B$, we infer from (2.8) that there is $i_{0} \in\{1, \ldots, l\}$ for which

$$
\mathcal{H}^{s}\left(B_{i_{0}} \cap B(x, r)\right)>2^{-1} l^{-1} r^{s} .
$$

Let $t=\max \{5 / \alpha, t(\eta)\}$, choose $q=(n, \eta)$ as in the proof of Theorem 2.4 and define $0<\varepsilon<1$ so that

$$
4^{-m} 2^{-1} l^{-1} \varepsilon^{m}-(q-1) 2^{s+1} t^{s} \varepsilon^{s}=4^{-m-1} l^{-1} \varepsilon^{m},
$$

recall that $s>m$ so that this is possible. Since the set $\left(V_{i_{0}}^{\perp}+\{x\}\right) \cap B(x, r)$ may be covered by $4^{m} \varepsilon^{-m}$ balls of radius $\varepsilon r$, there exists $y \in\left(V_{i_{0}}^{\perp}+\{x\}\right) \cap B(x, r)$ such that

$$
\mathcal{H}^{s}\left(B_{i_{0}} \cap B(x, r) \cap P_{V_{i_{0}}^{\perp}}^{-1}(B(y, \varepsilon r))\right)>4^{-m} 2^{-1} l^{-1} \varepsilon^{m} r^{s} .
$$

We now argue as in the proof of Theorem 2.4 above. We first observe that the slice $S=B_{i_{0}} \cap B(x, r) \cap P_{V_{i_{0}}^{\perp}}^{-1}(B(y, \varepsilon r))$ may be covered by $c_{1}^{-1} \varepsilon^{m-n}$ balls of radius $\varepsilon r$ for a constant $c_{1}=c_{1}(n, m)>0$. Then we use (2.11), (2.7), and (2.10) to find points $\left\{x_{1}, \ldots, x_{q}\right\} \in S$ such that $\left|x_{i}-x_{j}\right| \geq t \varepsilon r$ whenever $i \neq j$ and

$$
\begin{aligned}
\mathcal{H}^{s}\left(S \cap B\left(x_{i}, \varepsilon r\right)\right) & >c_{1} \varepsilon^{n-m}\left(4^{-m} 2^{-1} l^{-1} \varepsilon^{m} r^{s}-(q-1) 2^{s+1} t^{s} \varepsilon^{s} r^{s}\right) \\
& =c_{2}(3 r)^{s}
\end{aligned}
$$

for all $i$. Here $c_{2}=c_{2}(n, m, s, \alpha, \eta)=c_{1} 3^{-s} 4^{-m-1} l^{-1} \varepsilon^{m}$. Now the same geometric argument as in the proof of Theorem 2.4 implies that there is a point $z \in\left\{x_{1}, \ldots, x_{q}\right\}$ such that for each $\theta \in S^{n-1}$ we may find $w \in\left\{x_{0}, \ldots, x_{q}\right\} \backslash\{z\}$ so that

Also, since

$$
B(w, \varepsilon r) \subset B(z,(2+\varepsilon) r) \backslash(H(z, \theta, \eta) \cap B(z, 4 \varepsilon r / \alpha)) .
$$

$$
P_{V_{i_{0}}^{\perp}}^{-1}(B(y, \varepsilon r)) \cap B(z, 3 r) \backslash B(z, 4 \varepsilon r / \alpha) \subset X\left(z, 3 r, V_{i_{0}}, \alpha / 2\right),
$$


(see Figure $\complement$ ), we get

$$
\inf _{\theta \in S^{n-1}} \mathcal{H}^{s}\left(B \cap X\left(z, 3 r, V_{i_{0}}, \alpha / 2\right) \backslash H(z, \theta, \eta)\right) \geq c_{2}(3 r)^{s}
$$

by (2.12). Now $z \in B_{i_{0}}$ and we conclude, using (2.9), that $c \geq c_{2}=c_{2}(n, m, s, \alpha, \eta)$. This completes the proof.

Remark 2.6. Inspecting the proofs, one can read explicit expressions for the constants in Theorems 2.4 and 2.5. In Theorem 2.4, one gets $c \geq 2^{c_{1} /\left(-s \eta^{n-1}\right)}$ and in Theorem 2.5. one obtains $c \geq \alpha^{c_{3} /(s-m)} 2^{c_{2} /\left((m-s) \eta^{n-1}\right)}$. The constants $0<$ $c_{1}, c_{2}, c_{3}<\infty$ here depend only on $n$. The estimates obtained in this way are probably rather far from being optimal, although the best values are not known.

Our method can also be applied in a more general setting. A similar proof as above gives the following result. If $\mu$ is a measure on $\mathbb{R}^{n}, h:\left(0, r_{0}\right) \rightarrow(0, \infty)$, and $x \in \mathbb{R}^{n}$, we define $\bar{D}(\mu, x)$ and $\underline{D}(\mu, x)$ as the lower and upper limits, respectively, of the ratio $\mu(B(x, r)) / h(r)$ as $r \downarrow 0$.

Theorem 2.7. Suppose $0 \leq m<n$ and $h:\left(0, r_{0}\right) \rightarrow(0, \infty)$ is a function with

$$
\frac{h(\varepsilon r)}{\varepsilon^{m} h(r)} \longrightarrow 0 \quad \text { uniformly for all } 0<r<r_{0}
$$

as $\varepsilon \downarrow 0$. Let $\mu$ be a measure on $\mathbb{R}^{n}$ with $\bar{D}(\mu, x)<\infty$ for $\mu$-almost all $x \in \mathbb{R}^{n}$. For every $0<\alpha, \eta \leq 1$, there is a constant $c=c(n, m, h, \alpha, \eta)>0$ such that

$$
\limsup _{r \downarrow 0} \inf _{\substack{\theta \in S n-1 \\ V \in G(n, n-m)}} \frac{\mu(X(x, r, V, \alpha) \backslash H(x, \theta, \eta))}{h(r)} \geq c \bar{D}(\mu, x)
$$

for $\mu$-almost every $x \in \mathbb{R}^{n}$.

Let us make few comments related to the above theorem. Suppose that $h$ fulfills condition (2.13). Let $\mathcal{H}_{h}$ be the generalized Hausdorff measure which is constructed using $h$ as a gauge function; see [12, §4.9]. If $\mu=\left.\mathcal{H}_{h}\right|_{A}$, where $\mathcal{H}_{h}(A)<\infty$, then $\bar{D}(\mu, x)<\infty$ for $\mu$-almost every $x \in \mathbb{R}^{n}$, and thus Theorem 2.7 can be applied.

There are many natural gauge functions, such as $h(r)=r^{s} \log (1 / r)$ where $m<$ $s<n$, which satisfy (2.13). However, some interesting cases, such as $h(r)=$ $r^{m} / \log (1 / r)$, are not covered by this condition.

It seems to be unknown whether a similar result as Theorem 2.7 holds if one replaces the condition $\bar{D}(\mu, x)<\infty$ by $\underline{D}(\mu, x)<\infty$. The most interesting example falling into this category is obtained when $\mu=\left.\mathcal{P}^{s}\right|_{A}$ and $h(r)=r^{s}$, where $\mathcal{P}^{s}(A)<$ $\infty$ and $m<s<n$. Here $\mathcal{P}^{s}$ denotes the $s$-dimensional packing measure; see [12, $\S 5.10]$. See also Suomala [16] for related theorems.

\section{Sets With LARGe $k$-POROSity}

Mattila 11] proved Theorem 2.5 in the case $m=n-1$. Using this, he obtained the desired dimension bounds for 1-porous sets; see (1.3). Our result for $k$-porous sets follows applying a similar argument. 
For $\sqrt{2}-1<\varrho<\frac{1}{2}$ we define

$$
\begin{aligned}
t(\varrho) & =\frac{1}{\sqrt{1-2 \varrho}}, \\
\delta(\varrho) & =\frac{1-\varrho-\sqrt{\varrho^{2}+2 \varrho-1}}{\sqrt{1-2 \varrho}} .
\end{aligned}
$$

Notice that $\delta(\varrho) \rightarrow 0$ as $\varrho \rightarrow \frac{1}{2}$.

Lemma 3.1. Suppose $x \in \mathbb{R}^{n}, r>0, \sqrt{2}-1<\varrho<\frac{1}{2}, t=t(\varrho)$, and $\delta=\delta(\varrho)$. If $z \in \mathbb{R}^{n} \backslash\{x\}$ is such that $B(z$, etr $) \subset B(x, t r)$, then

$$
H(x+\delta r \theta, \theta) \cap B(x, r) \subset B(z, \varrho t r),
$$

where $\theta=(z-x) /|z-x|$.

Proof. To simplify the notation, we assume $r=1, x=0$, and $\theta=e_{1}=(1,0, \ldots, 0)$. This will not affect the generality. Let $y \in B(0,1) \backslash B(z, \varrho t)$. We have to show that

$$
y \notin H(x+\delta \theta, \theta) .
$$

By the Pythagorean Theorem we have

$$
\left|z-y_{1}\right|=\sqrt{|z-y|^{2}-\left|y-y_{1}\right|^{2}} \geq \sqrt{(\varrho t)^{2}-1} .
$$

Using this, we obtain

$$
y_{1}=|z|-\left|z-y_{1}\right| \leq t-\varrho t-\sqrt{(\varrho t)^{2}-1}=\delta,
$$

which implies (3.1).

Theorem 3.2. Suppose $0<k \leq n$. Then

$$
\sup \left\{s>0: \operatorname{por}_{k}(A)>\varrho \text { and } \operatorname{dim}_{\mathrm{H}}(A)>s \text { for some } A \subset \mathbb{R}^{n}\right\} \longrightarrow n-k
$$

as $\varrho \rightarrow \frac{1}{2}$.

Proof. Assume on the contrary that there exists $s>n-k$ such that for each $\sqrt{2}-1<\varrho<\frac{1}{2}$ there is a set $A_{\varrho}$ for which $\operatorname{dim}_{\mathrm{H}}\left(A_{\varrho}\right)>s$ and $\operatorname{por}_{k}\left(A_{\varrho}\right)>\varrho$. Take $\sqrt{2}-1<\varrho<\frac{1}{2}$ and such a set $A_{\varrho}$. Now $A_{\varrho}$ has a subset $B$ for which $\operatorname{dim}_{\mathrm{H}}(B)>s$ and $\operatorname{por}_{k}(B, x, r)>\varrho$ for all $x \in B$ and $0<r<r_{0}$ with some $r_{0}>0$. Clearly, the closure of $B$ also satisfies these conditions. Thus there is a closed set $F \subset \bar{B}$ (for example, use [2, Theorem 5.4]) such that $0<\mathcal{H}^{s}(F)<\infty$ and

$$
\operatorname{por}_{k}(F, x, r)>\varrho \quad \text { for all } x \in F \text { and } 0<r<r_{0} .
$$

Therefore, for any $x \in F$ and $0<r<r_{0} / t$, there are $z_{1}, \ldots, z_{k} \in \mathbb{R}^{n}$ such that $B\left(z_{i}, \varrho t r\right) \subset B(x, t r) \backslash F$ for $i=1, \ldots, k$, and $\left(z_{i}-x\right) \cdot\left(z_{j}-x\right)=0$ for $i \neq j$. Put $\theta_{i}=\left(z_{i}-x\right) /\left|z_{i}-x\right|$. Now applying Lemma 3.1 we have $H\left(x+\delta r \theta_{i}, \theta_{i}\right) \cap B(x, r) \subset$ $B\left(z_{i}, \varrho t r\right)$ for every $i$. Here $t=t(\varrho)$ and $\delta=\delta(\varrho)$. Thus

$$
F \cap B(x, r) \subset \bigcap_{i=1}^{k} B(x, r) \backslash H\left(x+\delta r \theta_{i}, \theta_{i}\right) .
$$

Put $\theta=-\frac{1}{\sqrt{k}} \sum_{i=1}^{k} \theta_{i}$ and take $V \in G(n, k)$ such that $\theta_{i} \in V$ for every $i$. Now choosing $\alpha$ and $\eta$ small enough, we have, using (3.2), that

$$
F \cap X(x, r, V, \alpha) \backslash H(x, \theta, \eta) \subset B\left(x, 2 n^{1 / 2} \delta r\right) .
$$




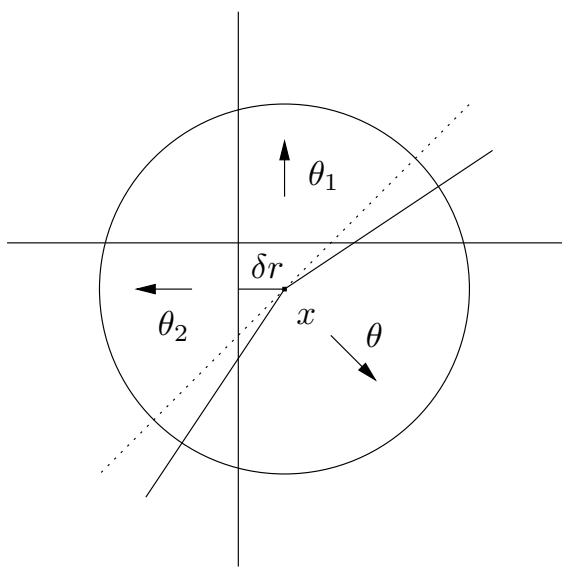

Figure D. Illustration for the proof of Theorem 3.2 the situation when $n=2$ and $k=2$.

Observe that the choice of $\alpha$ and $\eta$ does not depend on $\delta$ and hence nor on $\varrho$. Figure D illustrates the situation. Using Theorem 2.5. we may fix $x \in F$ and $0<r<r_{0} / t$ for which

$$
\mathcal{H}^{s}(F \cap X(x, r, V, \alpha) \backslash H(x, \theta, \eta)) \geq c 2^{2 s+1} n^{s / 2} r^{s},
$$

where $c=c(n, k, s, \alpha, \eta)>0$. By (1.1) we may assume that also

$$
\mathcal{H}^{s}\left(F \cap B\left(x, 2 n^{1 / 2} \delta r\right)\right) \leq 2^{2 s+1} n^{s / 2} \delta^{s} r^{s} .
$$

Combining (3.3)-(3.5), we have $c 2^{2 s+1} n^{s / 2} r^{s} \leq 2^{2 s+1} n^{s / 2} \delta^{s} r^{s}$ and hence

$$
s \leq \frac{\log c}{\log \delta(\varrho)} \text {. }
$$

But the constant $c$ does not depend on $\varrho$, and thus $\log c / \log \delta(\varrho) \rightarrow 0$ as $\varrho \rightarrow \frac{1}{2}$ giving a contradiction.

\section{Sets with Small Porosity}

Finally, let us briefly discuss the situation when porosity is small. The proof of the following theorem can be found for example in Martio and Vuorinen [10. We shall give here a different proof, and then show how the theorem can be improved when more information on the location of the holes is given.

Theorem 4.1. Let $A \subset \mathbb{R}^{n}$ be bounded and suppose that $\operatorname{por}_{1}(A, x, r) \geq \varrho$ for all $x \in A$ and $0<r<r_{0}$. Then $\operatorname{dim}_{\mathrm{M}}(A)<n-c \varrho^{n}$, where $c>0$ depends only on $n$.

Proof. We may assume that $r_{0}=1$ and $A \subset[0,1]^{n}$. Let us denote by $\mathcal{Q}_{j}$ the collection of all closed dyadic cubes $Q \subset[0,1]^{n}$ with side length $2^{-j}$. Let $l$ be the smallest integer with $2^{-l+2}<\varrho / \sqrt{n}$. It is easy to see that for any $Q \in \mathcal{Q}_{j}$ there is $Q^{\prime} \in \mathcal{Q}_{j+l}$ such that $Q^{\prime} \subset Q$ and $Q^{\prime} \cap A=\emptyset$. Let us fix one such $Q^{\prime}$ for each $Q \in \bigcup_{j=1}^{\infty} \mathcal{Q}_{j}$. Next we define a set $B \subset[0,1]^{n}$ by setting

$$
B=[0,1]^{n} \backslash \bigcup_{j=0}^{\infty} \bigcup_{Q \in \mathcal{Q}_{j}} Q^{\prime} .
$$




\begin{tabular}{|c|c|c|c|}
\hline \multicolumn{3}{|c|}{$f_{1}$} & $f_{2}$ \\
\hline \multicolumn{2}{|c|}{$f_{4}$} & $f_{5}$ & \multirow{3}{*}{$f_{3}$} \\
\hline$f_{7}$ & $f_{8}$ & \multirow{2}{*}{$f_{6}$} & \\
\hline & $f_{9}$ & & \\
\hline
\end{tabular}

Figure E. Similitudes $f_{k}$ in the proof of Theorem 4.1 when $n=2$ and $l=3$.

For any $Q \in \mathcal{Q}_{j}$, let $x_{Q}$ be the corner of $Q$ which is nearest to the origin, and let $\widetilde{Q}=\left\{x_{Q}\right\}+\left[0,2^{-j-1}\right]^{n}$. If we define $E \subset[0,1]^{n}$ by setting

$$
E=[0,1]^{n} \backslash \bigcup_{j=0}^{\infty} \bigcup_{Q \in \mathcal{Q}_{j}} \operatorname{int} \widetilde{Q}
$$

where int denotes the interior of a given set, then obviously $\operatorname{dim}_{M}(E) \geq \operatorname{dim}_{M}(B)$; see also [7. The set $E$ is the limit set of the iterated function system defined by the similitudes $f_{k}, k \in\left\{1,2, \ldots, l\left(2^{n}-1\right)\right\}$; see Figure E For any $i \in\{1, \ldots, l\}$, there are $2^{n}-1$ similitudes among $\left\{f_{k}\right\}_{k=1}^{l\left(2^{n}-1\right)}$ with contraction ratio $2^{-i}$. Since the open set condition is clearly satisfied, the dimension $s=\operatorname{dim}_{\mathrm{M}}(E)=\operatorname{dim}_{\mathrm{H}}(E)$ is given by

$$
\left(2^{n}-1\right) \sum_{i=1}^{l} 2^{-i s}=1
$$

see Hutchinson [3, §5]. This reduces to

$$
2^{n-s}=1+\left(2^{n}-1\right) 2^{-(l+1) s}
$$

and since $\log _{2}(1+x) \geq x /((1+x) \log 2)$ for $x \geq 0$, we have

$$
\begin{aligned}
s & =n-\log _{2}\left(1+\left(2^{n}-1\right) 2^{-(l+1) s}\right) \\
& \leq n-\log _{2}\left(1+\left(1-2^{-n}\right) 2^{-l n}\right) \\
& \leq n-\frac{2}{5 \log 2} 2^{-l n} \leq n-c \varrho^{n},
\end{aligned}
$$

where $c=(2 /(5 \log 2)) 2^{-3 n} n^{-n / 2}$. Because $A \subset B$ and $\operatorname{dim}_{\mathrm{M}}(B) \leq \operatorname{dim}_{\mathrm{M}}(E)=s$, we conclude that also $\operatorname{dim}_{\mathrm{M}}(A) \leq n-c \varrho^{n}$.

In the above proof, the use of the self-similar set $E$ is not a necessity, but it concretizes the situation. The key point in the proof is that for any cube $Q \subset \mathbb{R}^{n}$ which is small enough, one can find subcubes $Q_{1}, \ldots, Q_{l\left(2^{n}-1\right)} \subset Q$ such that 
$A \cap Q \subset \bigcup_{i=1}^{l\left(2^{n}-1\right)} Q_{i}$ and $\sum_{i=1}^{l\left(2^{n}-1\right)} \operatorname{diam}\left(Q_{i}\right)^{s}=\operatorname{diam}(Q)^{s}$, where $s$ is given by (4.2). From this the desired dimension bound follows easily.

Remark 4.2. In a sense the above result is the best possible one. There is a constant $c^{\prime}=c^{\prime}(n)>0$ and sets $A_{\varrho}, 0<\varrho<1 / 2$, with $\operatorname{dim}_{\mathrm{H}}\left(A_{\varrho}\right)>n-c^{\prime} \varrho^{n}$, and $\operatorname{por}_{1}\left(A_{\varrho}, x, r\right) \geq \varrho$ for all $r>0$ and $x \in \mathbb{R}^{n}$. See, for example, Koskela and Rohde [9], or estimate the Hausdorff dimension of the set $E$ from below.

Theorem 4.3. Let $A \subset \mathbb{R}^{n}$ be bounded and suppose that there is $V \in G(n, m)$ such that for all $x \in A$ and $0<r<r_{0}$ one has

$$
\sup \left\{\varrho^{\prime}: B\left(z, \varrho^{\prime} r\right) \subset B(x, r) \backslash A \text { for some } z \in V+\{x\}\right\} \geq \varrho .
$$

Then $\operatorname{dim}_{\mathrm{M}}(A)<n-c \varrho^{m}$, where $c>0$ depends only on $n$ and $m$.

Proof. Without losing the generality we may assume that $V=\mathbb{R}^{m}=\left\{x \in \mathbb{R}^{n}\right.$ : $\left.x_{m+1}=x_{m+2}=\ldots=x_{n}=0\right\}, r_{0}=\sqrt{n}$, and $A \subset[0,1]^{n}$. Let $\mathcal{Q}_{j}$ be, as before, the collection of all closed dyadic cubes $Q \subset[0,1]^{n}$ with side length $2^{-j}$, and let $\widetilde{\mathcal{Q}}_{j}=\left\{P_{V}(Q): Q \in \mathcal{Q}_{j}\right\}$ and $\mathcal{Q}_{j}^{\prime}=\left\{P_{V^{\perp}}(Q): Q \in \mathcal{Q}_{j}\right\}$. Here $P_{V}$ is the orthogonal projection onto $V$. Furthermore, let $l$ be the smallest integer with $2^{-l+2}<\varrho / \sqrt{n}$.

We define a set $E=E_{l, m} \subset V$ as in the proof of Theorem 4.1. For $j \in \mathbb{N}$ we let $a_{j}=a_{j, l, m}$ denote the minimum number of cubes from the collection $\widetilde{\mathcal{Q}}_{j}$ that are needed to cover $E$. The proof of Theorem 4.1 yields that

$$
\lim _{j \rightarrow \infty} \frac{\log a_{j}}{\log \left(2^{j}\right)} \leq m-c 2^{-m l},
$$

where $c>\frac{1}{2}$ is an absolute constant.

It is straightforward to convince oneself of the following fact: If $\widetilde{Q} \in \widetilde{\mathcal{Q}}_{j}$ and $Q^{\prime} \in \mathcal{Q}_{j+l}^{\prime}$, then there is $Q \in \mathcal{Q}_{j+l}$ such that $P_{V^{\perp}}(Q)=Q^{\prime}, P_{V}(Q) \subset \widetilde{Q}$, and $A \cap Q=\emptyset$. From this observation it follows that given $Q^{\prime} \in \mathcal{Q}_{j}^{\prime}$, only $a_{j}$ cubes from the collection $\left\{Q \in \mathcal{Q}_{j}: P_{V^{\perp}}(Q)=Q^{\prime}\right\}$ touch the set $A$. Thus only $2^{j(n-m)} a_{j}$ cubes from the collection $\mathcal{Q}_{j}$ are needed to cover $A$. Using (4.4), we calculate

$$
\begin{aligned}
\operatorname{dim}_{\mathrm{M}}(A) & \leq \limsup _{j \downarrow 0} \frac{\log \left(2^{j(n-m)} a_{j}\right)}{\log \left(2^{j}\right)}=n-m+\limsup _{j \downarrow 0} \frac{\log a_{j}}{\log \left(2^{j}\right)} \\
& \leq n-c 2^{-m l} \leq n-c 2^{-3 m} n^{-m / 2} \varrho^{m} .
\end{aligned}
$$

The proof is finished.

Remark 4.4. Suppose that $V \in G(n, m)$ is fixed and $A \subset \mathbb{R}^{n}$ is such that (4.3) holds for every $x \in A$ and $0<r<r_{x}$, where $r_{x}>0$ depends on the point $x$. It follows immediately from Theorem 4.3 that $\operatorname{dim}_{H}(A) \leq \operatorname{dim}_{\mathrm{p}}(A) \leq n-c \varrho^{m}$, where $c$ is as in Theorem 4.3 and $\operatorname{dim}_{\mathrm{p}}$ denotes the packing dimension; see [12, $\S 5.9$ ]. The above dimension estimates are also sharp. Consider, for example, sets of the form $E \times \mathbb{R}^{n-m}$, where $E \subset \mathbb{R}^{m}$ is as in the proof of Theorem 4.1.

Remark 4.5. After the submission of this article in May 2004, there has been considerable progress in the study of conical densities and porosities. Most notably, the question posed after Theorem 2.7 has been answered positively in [8]. For improvements of Theorems 3.2 and 4.1, see [6] and [5], respectively. 


\section{ACKNOWLEDGMENTS}

The authors are indebted to Professor Pertti Mattila for his valuable comments on the manuscript. The authors also thank Esa Järvenpää, Maarit Järvenpää, Pekka Koskela, Tomi Nieminen, Kai Rajala and Eero Saksman for useful discussions during the preparation of this article.

\section{REFERENCES}

[1] P. Erdős and Z. Füredi. The greatest angle among $n$ points in the $d$-dimensional Euclidean space. North-Holland Math. Stud., 75:275-283, 1983. MR0841305 (87g:52018)

[2] K. J. Falconer. Geometry of Fractal Sets. Cambridge University Press, Cambridge, 1985. MR867284 (88d:28001)

[3] J. E. Hutchinson. Fractals and self-similarity. Indiana Univ. Math. J., 30(5):713-747, 1981. MR625600 (82h:49026)

[4] E. Järvenpää and M. Järvenpää. Porous measures on $\mathbb{R}^{n}$ : Local structure and dimensional properties. Proc. Amer. Math. Soc., 130(2):419-426, 2002. MR.1862121 (2002h:28001)

[5] E. Järvenpää, M. Järvenpää, A. Käenmäki, T. Rajala, S. Rogovin, and V. Suomala. Packing dimension and Ahlfors regularity of porous sets in metric spaces. Math. Z., 266(1):83-105, 2010.

[6] E. Järvenpää, M. Järvenpää, A. Käenmäki, and V. Suomala. Asympotically sharp dimension estimates for $k$-porous sets. Math. Scand., 97(2):309-318, 2005. MR2191709 (2006j:28016)

[7] A. Käenmäki. On natural invariant measures on generalised iterated function systems. Ann. Acad. Sci. Fenn. Math, 29(2):419-458, 2004. MR2097242 (2005g:37044)

[8] A. Käenmäki and V. Suomala. Conical upper density theorems and porosity of measures. Adv. Math., 217(3):952-966, 2008. MR2383891

[9] P. Koskela and S. Rohde. Hausdorff dimension and mean porosity. Math. Ann., 309:593-609, 1997. MR1483825 (98k:28004)

[10] O. Martio and M. Vuorinen. Whitney cubes, $p$-capacity, and Minkowski content. Exposition. Math., 5(1):17-40, 1987. MR880256 (88e:28004)

[11] P. Mattila. Distribution of sets and measures along planes. J. London Math. Soc. (2), 38(1):125-132, 1988. MR949087 (89f:28019)

[12] P. Mattila. Geometry of Sets and Measures in Euclidean Spaces: Fractals and Rectifiability. Cambridge University Press, Cambridge, 1999. MR.1333890 (96h:28006)

[13] A. Salli. Upper density properties of Hausdorff measures on fractals. Ann. Acad. Sci. Fenn. Ser. A I Math. Dissertationes No. 55, 1985. MR809031 (87c:28019)

[14] A. Salli. On the Minkowski dimension of strongly porous fractal sets in $\mathbb{R}^{n}$. Proc. London Math. Soc. (3), 62(2):353-372, 1991. MR.1085645 (91m:28008)

[15] J. Sarvas. The Hausdorff dimension of the branch set of a quasiregular mapping. Ann. Acad. Sci. Fenn. Ser. A I Math., 1(2):297-307, 1975. MR0396945(53:805)

[16] V. Suomala. On the conical density properties of measures on $\mathbb{R}^{n}$. Math. Proc. Cambridge Philos. Soc., 138(3):493-512, 2005. MR2138576 (2006f:28006)

[17] D. A. Trocenko. Properties of regions with a nonsmooth boundary. Sibirsk. Mat. Zh., 22(4):221-224, 1981. MR624419 (82h:30015)

[18] J. Väisälä. Porous sets and quasisymmetric maps. Trans. Amer. Math. Soc., 299(2):525-533, 1987. MR869219(88a:30049)

Department of Mathematics and Statistics, P.O. Box 35 (MaD), FI-40014 University OF JYVÄSKYlä, FinLAND

E-mail address: antti.kaenmaki@jyu.fi

Department of Mathematics and Statistics, P.O. Box 35 (MaD), FI-40014 University OF JYVÄSKYLÄ, FinLAND

E-mail address: ville.suomala@jyu.fi 